\title{
LXI. On a general theorem respecting electrical influence
}

\section{Professor R. Clausius}

To cite this article: Professor R. Clausius (1877) LXI. On a general theorem respecting electrical influence, Philosophical Magazine Series 5, 4:27, 454-458, DOI: 10.1080/14786447708639368

To link to this article: http://dx.doi.org/10.1080/14786447708639368

曲 Published online: 13 May 2009.

Submit your article to this journal $\pi$

Џ Article views: 4

Q View related articles $\sqsubset$ 


\section{[ 454$]$}

LXI. On a General Theorem respecting Electrical Influence. By Professor R. Claudsius*.

CERTAIN reciprocity-theorems respecting the reciprocal $\mathcal{U}$ influence of two conductors of electricity have already been repeatedly advanced by various authors. I take leave to communicate here a very general, and, so far as I know, new theorem, from which several of those theorems result as immediate consequences.

Given any number whatever of conducting bodies $\mathrm{C}_{1}, \mathrm{C}_{2}, \mathrm{C}_{3}$, $\& c$., which act by way of influence on each other. These are to be charged with electricity in two different ways. In the first charge let the quantities of electricity present upon the individual bodies be

$\mathrm{Q}_{1}, \mathrm{Q}_{2}, \quad \mathrm{Q}_{3}$, \&..,

and the levels of potential thereby produced on the bodies

$$
\mathrm{V}_{1}, \quad \mathrm{~V}_{2}, \mathrm{~V}_{3}, \text { \&c.; }
$$

and in the second charge let the quantities of electricity and the potential-levels be

$$
\begin{array}{lll}
\mathfrak{Q}_{1}, & \mathfrak{Q}_{2}, & \mathfrak{Q}_{3}, \quad \& c ., \\
\mathfrak{V}_{1}, & \mathfrak{V}_{2}, & \mathfrak{V}_{3}, \quad \& c .
\end{array}
$$

Then the following equation holds good:-

$$
\mathrm{V}_{1} \mathfrak{Q}_{1}+\mathrm{V}_{2} \mathfrak{Q}_{2}+\mathrm{V}_{3} \mathfrak{Q}_{3}+\& c .=\mathfrak{V}_{1} \mathrm{Q}_{1}+\mathfrak{V}_{2} \mathrm{Q}_{2}+\mathfrak{B}_{3} \mathrm{Q}_{3}+\& \mathrm{c} .
$$

or, employing summation-symbols, more briefly written,

$$
\Sigma V \mathfrak{Q}=\Sigma \mathfrak{B} \mathfrak{Q} \text {. . . . . . . }
$$

For the proof of this equation, let us imagine an infinitely large spherical surface formed round a point situated in the vicinity of the bodies, and to the infinite space lying between the bodies and the spherical surface apply the well-known equation of Green, while we denote the two functions therein occurring by $\mathrm{V}$ and $\mathfrak{B}$, understanding by these the potentialfunctions corresponding to the first and second charges respectively. The equation will then read:-

$$
\int \mathrm{V} \frac{\partial \mathfrak{B}}{\partial n} d \omega+\int \mathrm{V} \Delta \mathfrak{B} d \tau=\int \mathfrak{B} \frac{\partial \mathrm{V}}{\partial n} d \omega+\int \mathfrak{V} \Delta \mathrm{V} d \tau .
$$

Here $d \omega$ signifies an element of the surface which bounds the space we are considering, and which consists of the surfaces of the given bodies and the infinitely large surface of the sphere; $n$ is to represent the normal erected upon the surfaceelement (reckoned positive in the direction of the space considered); and the integrals containing $d \omega$ refer to the total limiting surface. Futher, $d \tau$ denotes an element of the space

* Translated from a separate impression, communicated by the Author, from Poggendorff's Annalen, new series, vol. i. pp. 493-499. 
considered, by $\Delta$ is signified the operation $\frac{\partial^{2}}{\partial x^{2}}+\frac{\partial^{2}}{\partial y^{2}}+\frac{\partial^{2}}{\partial z^{2}}$, and the integrals which contain $d \tau$ are to be extended over the entire space under consideration.

But now, with both the adopted methods of charging, no electricity is contained in this space; and consequently there hold good in it everywhere the equations

$$
\Delta \mathrm{V}=0 \text { and } \Delta \mathfrak{B}=0 \text {, }
$$

by which equation (1) reduces to

$$
\int \mathrm{V} \frac{\partial \mathfrak{B}}{\partial n} d \omega=\int \mathfrak{B} \frac{\partial \mathrm{V}}{\partial n} d \omega . \quad . \quad . \quad, .
$$

In this equation also occurs a simplification. The two integrals are, according to the above, to be taken over the surfaces of the given bodies and over the spherical surface. But now, if $\mathrm{R}$ signifies the infinite radius of the spherical surface, the values of $\mathrm{V}$ and $\mathfrak{B}$ on that surface are infinitesimal quantities of the order $\frac{1}{R}$, and the values of the differential coefficients $\frac{\partial V}{\partial n}$ and $\frac{\mathfrak{B}}{\partial n}$ at which the direction of the normal coincides with that of the radius are infinitely small quantities of the order $\frac{1}{\mathrm{R}^{2}}$. The products $V \frac{\partial \mathfrak{B}}{\partial n}$ and $\mathfrak{B} \frac{\partial \mathrm{V}}{\partial n}$ are consequently, on the spherical surface, infinitesimal quantities of the order $\frac{1}{\mathrm{R}^{3}}$. As for the surface-element of the spherical surface, we can replace it by the product $\mathrm{R}^{2} d \sigma$, if $d \sigma$ denotes an element of the solid angle at the centre of the spherical surface. Then the factor, on both sides of the equation, with which $d \sigma$ under the integral-symbol is affected is an infinitesimal one of the order $\frac{1}{\mathrm{R}}$, whence it follows that, of both integrals, the part which refers to the spherical surface, and which is to be taken according to $\sigma$ from 0 to $4 \pi$, is infinitely small and may be neglected. Consequently the integrals in equation (2) need only to be referred to the surfaces of the given bodies.

On the surface of each body the potential-function is constant; hence, for the part of the integral which refers to it, it can be taken out of the symbol of the integral. Accordingly we can write equation (2) thus :-

$$
\left.\begin{array}{rl} 
& V_{1} \int \frac{\partial \mathfrak{B}}{\partial n} d \omega_{1}+V_{2} \int \frac{\partial \mathfrak{B}}{d n} d \omega_{2}+V_{3} \int \frac{\partial \mathfrak{B}}{\partial n} d \omega_{3}+\& c . \\
= & \mathfrak{V}_{1} \int \frac{\partial \mathrm{V}}{\partial n} d \omega_{1}+\mathfrak{B}_{2} \int \frac{\partial V}{\partial n} d \omega_{2}+\mathfrak{B}_{3} \int \frac{\partial \mathrm{V}}{\partial n} d \omega_{3}+\& \mathrm{c}_{2},
\end{array}\right\} .
$$


in which $d \omega_{1}, d \omega_{2}, d \omega_{3}, \& c$. will be surface-elements of the bodies $\mathrm{C}_{1}, \mathrm{C}_{2}, \mathrm{C}_{3}$, \&c., and the different integrals are to be referred to the surfaces of the respective bodies.

Further, it is known that a simple relation subsists, on the surface of a conducting body charged with electricity, between the differential coefficient, taken in the direction of the normal, of the potential-function, and the electrical density. If, namely, $h$ and $b$ denote the surface-densities with the two charges, the equations

$$
\frac{\partial V}{\partial n}=-4 \pi h \text { and } \frac{\partial \mathfrak{V}}{\partial n}=-4 \pi \mathfrak{b}
$$

hold, and thereby (3) is transformed into

$$
\left.\begin{array}{rl} 
& V_{1} \int \mathfrak{b} d \omega_{1}+V_{2} \int \mathfrak{b} d \omega_{2}+V_{3} \int \mathfrak{b} d \omega_{3}+d c . \\
= & \mathfrak{V}_{1} \int h d \omega_{1}+\overline{\mathfrak{V}}_{2} \int h d \omega_{2}+\mathfrak{B}_{3} \int h d \omega_{3}+\& c .
\end{array}\right\} .
$$

The integrals occurring here again are, however, nothing more than the quantities of electricity present upon the respective bodies; and consequently we obtain the equation which was to be demonstrated :-

$$
\mathrm{V}_{1} \mathfrak{\Omega}_{1}+\mathrm{V}_{2} \mathfrak{\Omega}_{2}+\mathrm{V}_{3} \mathfrak{\Omega}_{3}+\& c .=\mathfrak{B}_{1} \mathrm{Q}_{1}+\mathfrak{B}_{2} \mathrm{Q}_{2}+\mathfrak{B}_{3} \mathrm{Q}_{3}+\& \mathrm{c} .
$$

Now the applications of this equation are facilitated by the fact that it can be still further simplified under certain often occurring conditions.

If, namely, we consider the terms which refer to any one of the given bodies, which may be called $\mathrm{C}_{i}$, viz. the two products

$$
\mathrm{V}_{i} \mathfrak{O}_{i} \text { and } \mathfrak{B}_{i} \mathrm{Q}_{i} \text {, }
$$

these become in two cases nil, so that they can be omitted from the equation. If the body is in conducting connexion with the earth, its potential-level remains at zero with every charge of the system; consequently we have to put for this case

$$
\nabla_{i}=\mathfrak{B}_{i}=0,
$$

through which the above products vanish. Further, when the body is insulated and initially unelectric, and receives, on charging, no electricity from without, but only through influence undergoes an unequal distribution of its own electrieity, then its surface becomes in part positively, and in part negatively electric, in such wise that the total electricity present on the surface remains nil. We have consequently then to put

$$
\mathrm{Q}_{i}=\mathfrak{\Omega}_{i}=0,
$$

through which the above products again vanish. Accordingly 
the following rule can be laid down:-Those bodies which during both charges are either in conducting communication with the earth, or insulated and initially unelectric, and in being charged receive no electricity, may be left out of consideration in constructing equation (I.).

It may now, as a special case, be assumed that with all the given bodies, with the exception of $\mathrm{C}_{1}$ and $\mathrm{C}_{2}$, one of the two conditions mentioned occurs. The equation then reduces to

$$
\mathrm{V}_{1} \mathfrak{Q}_{1}+\mathrm{V}_{2} \mathfrak{Q}_{2}=\mathfrak{B}_{1} \mathfrak{Q}_{1}+\mathfrak{B}_{2} \mathrm{Q}_{2}
$$

If this equation be still further simplified by making special assumptions respecting the behaviour of the bodies $\mathrm{C}_{1}$ and $\mathrm{C}_{2}$, we arrive at the reciprocity-theorems mentioned at the commencement.

We will first suppose the two bodies insulated and initially unelectric, and assume that in the first charging the body $\mathrm{C}_{1}$ only acquires the quantity of electricity $\mathrm{E}$, by the influence of which the potential-level $\mathrm{V}_{2}$ is produced in $\mathrm{C}_{2}$, and that in the second charging only the body $\mathrm{C}_{2}$ receives the quantity of electricity $\mathrm{E}$, through the influence of which the potentiallevel $\mathfrak{V}_{1}$ arises in $\mathrm{C}_{1} \ldots$ In this case we have to put

$$
\mathrm{Q}_{2}=\mathfrak{\Omega}_{1}=0, \quad \mathrm{Q}_{1}=\mathfrak{\Omega}_{2}=\mathrm{E},
$$

by which (5) is changed into

or

$$
\mathrm{V}_{2} \mathrm{E}=\mathfrak{B}_{1} \mathrm{E}
$$

$$
\mathrm{V}_{2}=\mathfrak{B}_{1} \text {. . . . . . . . }
$$

Consequently the potential-level which arises in $\mathrm{C}_{2}$ through $\mathrm{C}_{1}$ being charged with a certain amount of electricity, and that which arises in $\mathrm{C}_{1}$ through $\mathrm{C}_{2}$ being charged with the same amount, are equal to one another.

We will make the further assumption, that in the first charging $C_{1}$ is charged up to the potential-level $K$, while $C_{2}$ is connected to earth and through influence receives from the earth the amount of electricity $Q_{2}$, and that in the second charging $\mathrm{C}_{2}$ is charged up to the potential-level $\mathrm{K}$, while $\mathrm{C}_{1}$ is connected to earth and through influence receives the amount $\mathfrak{Q}_{1}$ of electricity. We have then to put

$$
\mathrm{V}_{2}=\mathfrak{V}_{1}=0, \quad \mathrm{~V}_{1}=\mathfrak{V}_{2}=\mathrm{K},
$$

whereby (5) is changed into

or

$$
\mathrm{K} \mathfrak{D}_{1}=\mathrm{K} \mathrm{Q}_{2} \text {, }
$$

$$
\mathfrak{Q}_{1}=Q_{2} \cdot \text {. . . . . . }
$$

Consequently the amount of electricity which through influ- 
ence is collected on $\mathrm{C}_{2}$ when $\mathrm{C}_{1}$ is charged up to a certain potential-level, and that which in the charging of $\mathrm{C}_{2}$ up to the same potential is through influence collected on $\mathrm{C}_{1}$, are equal the one to the other.

Beside these two conclusions, here cited as examples, referable to two very simple special cases, from equation (I.) many other similar inferences can of course be drawn.

LX. On the Potential of an Ellipsoid at an External Point. By Colonel A. R. Clarke, C.B., F.R.S.*

TN connexion with the subject of the attraction of the earth 1 upon external objects, there is a series which is usually brought forward by writers on astronomy, viz. that which results from expanding into a series in descending powers of $r$ the expression for $V$,

$$
\mathrm{V}=\iiint \frac{d x d y d z}{\left\{(f-x)^{2}+(g-y)^{2}+(h-z)^{2}\right\}^{\frac{1}{2}}}
$$

r being the distance $=\left(f^{2}+g^{2}+h^{2}\right)^{\frac{1}{2}}$ of the attracted external particle from the origin, $x, y, z$ the coordinates of any element of the mass, the density being unity throughout. Put $x^{2}+y^{2}+z^{2}=\rho^{2}, \alpha x+\beta y+\gamma z=\sigma$, where $\alpha, \beta, \gamma$ are the direction-cosines of $r$; then the radical in the expression for $V$ may be expanded in the form

$$
\frac{1}{r}+\frac{Q_{1}}{r^{2}}+\frac{Q_{2}}{r^{3}}+\frac{Q_{3}}{r^{4}}+\ldots,
$$

where $Q_{i}$ is a homogeneous function of $x, y, z$ of the degree $i$. When the body is an ellipsoid referred to its principal axes, the terms in which $i$ is odd will disappear in integrating over the volume of the ellipsoid; so that in this case, $\mathbf{M}$ being the mass of the ellipsoid,

$$
\mathrm{V}=\frac{\mathrm{M}}{r}+\frac{1}{r^{3}} \int \mathrm{Q}_{2} d m+\frac{1}{r^{5}} \int \mathrm{Q}_{4} d m+\frac{1}{r^{7}} \int \mathrm{Q}_{6} d m+\ldots,
$$

which is the series alluded to. Lagrange's investigation of the terms (as far as just written down) of this series is referred to in Todhunter's 'History of the Theory of Attraction and the Figure of the Earth,' vol. ii. p. 161. See also Thomson and Tait, 'Natural Philosophy,' pp. 401, 402 ; Pontécoulant, Théorie Analytique du Systeme du Monde, ii. p. 233, where the above expression for the potential is given as one suitable

* Communicated by the Author. 